\title{
Test of parity-conserving time-reversal invariance using polarized neutrons and nuclear spin aligned holmium
}

\author{
P. R. Huffman, * N. R. Roberson, and W. S. Wilburn \\ Physics Department, Duke University, Durham, North Carolina 27708-0305 \\ and Triangle Universities Nuclear Laboratory, Durham, North Carolina 27708-0308 \\ C. R. Gould, D. G. Haase, C. D. Keith,$^{\dagger}$ B. W. Raichle, M. L. Seely ${ }^{\ddagger}$ and J. R. Walston \\ Physics Department, North Carolina State University, Raleigh, North Carolina 27695-8202 \\ and Triangle Universities Nuclear Laboratory, Durham, North Carolina 27708-0308
}

(Received 28 October 1996)

\begin{abstract}
A test of parity-conserving, time-reversal noninvariance (PC TRNI) has been performed in $5.9 \mathrm{MeV}$ polarized neutron transmission through nuclear spin aligned holmium. The experiment searches for the $T$-violating fivefold correlation via a double modulation technique-flipping the neutron spin while rotating the alignment axis of the holmium. Relative cross sections for spin-up and spin-down neutrons are found to be equal to within $1.2 \times 10^{-5}$ ( $80 \%$ confidence). This is a two orders of magnitude improvement compared to traditional detailed balance studies of time reversal, and represents the most precise test of PC TRNI in a dynamical process, to our knowledge. [S0556-2813(97)06905-7]
\end{abstract}

PACS number(s): 24.80.+y, 24.70.+s, 25.40.Dn, 28.20.Cz

\section{INTRODUCTION}

Parity-conserving, time-reversal noninvariance (PC TRNI) is a second-order effect in the minimal standard model and its effects are expected to be extremely small [1-4]. Nevertheless, searches for PC TRNI in nuclear physics have a long and varied history, including studies of $\gamma$-ray decay correlations [5], energy-level spacings [6] and energy shifts [7], comparisons of polarization versus analyzing powers in nucleon-nucleon scattering [8,9], and comparisons of relative cross sections using detailed balance [10]. Previously, the most precise dynamical bound came from the detailed balance studies of the reaction ${ }^{24} \operatorname{Mg}(\alpha, p){ }^{27} \mathrm{Al}$ and its inverse, where relative differential cross sections were found to be equal to within $5.1 \times 10^{-3}$ (80\% confidence) [10].

In this article, we present results from an improved PC TRNI measurement using polarized neutron transmission through a rotating, cryogenically aligned, ${ }^{165} \mathrm{Ho}$ target $[11,12]$. The measurement tests reciprocity, or more specifically, the symmetry of the elastic scattering $S$ matrix. We find the relative cross sections for spin-up versus spin-down $5.9 \mathrm{MeV}$ neutrons to be equal to within $1.2 \times 10^{-5}(80 \%)$, a factor of 400 improvement in a measurement of a TRNI cross section.

Simonius has shown that for meson exchange models, PC TRNI arises through charged $\rho$ meson exchanges [13]. We take advantage of a recently developed model incorporating $T$-violating $\rho$ meson exchange [14] to make a more funda-

\footnotetext{
*Present address: Department of Physics, Harvard University, Cambridge, MA 02138.

${ }^{\dagger}$ Present address: Indiana University Cyclotron Facility, Bloomington, IN 47405.

FPresent address: Thomas Jefferson National Accelerator Facility, Newport News, VA 23606.
}

mental comparison of the results of our experiment with other low-energy nuclear physics measurements.

The remainder of this article is organized as follows. In Sec. II we discuss the TRNI observable being measured. Section III follows with a description of the experimental apparatus and the data acquisition procedures. Section IV discusses supplemental measurements needed to interpret the TRNI measurement. Section V estimates upper bounds on sources of false asymmetries. A data analysis section (VI) is presented before concluding with Sec. VII, which compares our results to tests of parity-conserving, time-reversal noninvariance in other systems. A brief version of this work has been published previously [15].

\section{THEORY}

The spin-dependent total cross section for neutron scattering has been discussed by numerous authors [16-21]. Following the notation introduced in reference [21], the neutron total cross section $\sigma_{T}$ can be written as a sum of partial cross sections $\sigma_{k K}$ that depend on the rank of the statistical tensors describing the polarization states of the beam $(k)$ and target $(K)$. The total cross section is given by

$$
\sigma_{T}=\sum_{k K} \widetilde{t}_{k 0}(s) \widetilde{t}_{K 0}(I) \sigma_{k K}
$$

where the beam direction $(\hat{\mathbf{k}})$ is taken along the $z$ axis, $s$ denotes the spin of the incident neutron beam (direction $\hat{\mathbf{s}}$ ), and $I$ denotes the spin of the target (direction $\hat{\mathbf{I}}$ ). The spinorbit angular momentum coupling scheme $(\ell+\mathbf{s}=\mathbf{j}$, $\mathbf{j}+\mathbf{I}=\mathbf{J})$ is used where $\ell$ is the orbital angular momentum of the neutron. The quantities $\widetilde{t_{k 0}}(s)$ and $\widetilde{t}_{K 0}(I)$ denote the polarization states of the beam and target in their axially symmetric frames. The partial cross sections, $\sigma_{k K}$, are 


$$
\begin{aligned}
\sigma_{k K}= & 4 \pi \chi^{2} \frac{\hat{k} \hat{K}}{\hat{s} \hat{I}} \operatorname{Im} \sum_{\Lambda} \hat{\Lambda} C_{k K \Lambda}(\hat{\mathbf{s}} \hat{\mathbf{I}}) \\
& \times \sum_{J \ell j \ell^{\prime} j^{\prime}}(2 J+1) \hat{\ell} \hat{J} \hat{J}^{\prime}\left\langle\ell \Lambda 00 \mid \ell^{\prime} 0\right\rangle \\
& \times W\left(J j I K ; I j^{\prime}\right)\left\{\begin{array}{ccc}
\ell & s & j \\
\Lambda & k & K \\
\ell^{\prime} & s & j^{\prime}
\end{array}\right\} T_{\ell^{\prime} j^{\prime} \ell j}^{J},
\end{aligned}
$$

where $\hat{a}=\sqrt{2 a+1}, \chi$ is the reduced wavelength, and the angular brackets, $W$, and the braces denote the ClebschGordon, Racah, and $9-j$ coefficients respectively. The $T$-matrix elements, $T_{\ell^{\prime} j^{\prime} \ell j}^{J}=(1 / 2 i)\left(S_{\ell^{\prime} j^{\prime} \ell j}^{J}-\delta_{\ell \ell^{\prime}} \delta_{j j^{\prime}}\right)$, contain the elements of the elastic-scattering $S$ matrix for the reaction, and the coefficients $C_{k K \Lambda}(\hat{\mathbf{s}} \hat{\mathbf{I}} \hat{\mathbf{k}})$ are defined by

$$
C_{k K \Lambda}(\hat{\mathbf{s}} \hat{\mathbf{k}} \hat{\mathbf{k}})=\frac{(4 \pi)^{3 / 2}}{\hat{k} \hat{K}}\left[\left[Y_{k}^{q}(\hat{\mathbf{s}}) \otimes Y_{K}^{-q}(\hat{\mathbf{I}})\right]_{\Lambda}^{0} \otimes Y_{\Lambda}^{0}(\hat{\mathbf{k}})\right]_{0}^{0},
$$

where the product of two spherical tensors $Y_{k}^{q}(\hat{\mathbf{s}})$ and $Y_{K}^{-q}(\hat{\mathbf{I}})$ coupled to $\Lambda 0$ is given by

$$
\left[Y_{k}^{q}(\hat{\mathbf{s}}) \otimes Y_{K}^{-q}(\hat{\mathbf{I}})\right]_{\Lambda}^{0}=\sum_{q} Y_{k}^{q}(\hat{\mathbf{s}}) Y_{K}^{-q}(\hat{\mathbf{I}})\langle k K q-q \mid \Lambda 0\rangle .
$$

We specialize this expression to the situation where the neutron spin direction $(\hat{\mathbf{s}})$ is along the $y$ axis, its momentum $(\hat{\mathbf{k}})$ is along the $z$ axis, and the nuclear-spin aligned target ( $K$ even) has its alignment axis in the $x-z$ plane. In this case, only three partial cross sections are important: the unpolarized cross section $\left(\sigma_{00}\right)$, the deformation effect cross section $\left(\sigma_{02}\right)$, and the fivefold correlation cross section $\left[\sigma_{12}(\Lambda=2) \equiv \sigma_{\mathrm{FC}}\right]$. The total cross section to order $K=2$ is

$$
\sigma_{T}=\sigma_{00}+\widetilde{t}_{20}(I) \sigma_{02}+\widetilde{t}_{10}(s) \widetilde{t}_{20}(I) \sigma_{\mathrm{FC}} .
$$

This experiment seeks to set a bound on $\sigma_{\mathrm{FC}}$, which is zero if time reversal is a good symmetry $\left(S_{\ell^{\prime} j^{\prime} \ell j}^{J}=S_{\ell j \ell^{\prime} j^{\prime}}^{J}\right)$.

The fivefold correlation (FC) term in the cross section depends on both the beam polarization and target alignment. The $T$-violating cross section $\sigma_{\mathrm{FC}}$ is given by

$$
\begin{aligned}
\sigma_{\mathrm{FC}}= & \pi \chi^{2} \frac{\sqrt{15}}{2 \hat{s}^{2} \hat{I}} \sin 2 \theta \sum_{J \ell j \ell^{\prime} j^{\prime}}(-1)^{s-\ell-j^{\prime}}(2 J+1) \hat{\ell} \hat{\jmath} \hat{J}^{\prime} \\
& \times \frac{\ell(\ell+1)-\ell^{\prime}\left(\ell^{\prime}+1\right)-j(j+1)+j^{\prime}\left(j^{\prime}+1\right)}{\sqrt{s(s+1)}} \\
& \times\left\langle\ell 200 \mid \ell^{\prime} 0\right\rangle W\left(J j I 2 ; I j^{\prime}\right) \\
& \times W\left(\ell j \ell^{\prime} j^{\prime} ; s 2\right) \operatorname{Im}\left\{i T_{\ell^{\prime} j^{\prime} \ell j}^{J}\right\},
\end{aligned}
$$

where $\theta$ is defined as the angle between the directions of the diagonal tensors describing the target alignment and the beam direction (i.e., $\hat{\mathbf{I}} \cdot \hat{\mathbf{k}}=\cos \theta$ ). For a nonzero effect, a transfer of two units of angular momentum is required $\left(\ell+2=\ell^{\prime}\right)$. A nonzero value of $\sigma_{\mathrm{FC}}$ implies the $S$ matrix is not symmetric and that reciprocity does not hold.

The fivefold correlation partial cross section exhibits a $\sin 2 \theta$ angular dependence and is isolated by forming an asymmetry between the angle-dependent transmission, $T^{+}$( $\theta$ ) for neutrons with spin-up and the angle-dependent transmission, $T^{-}(\theta)$ for neutrons with spin-down

$$
\epsilon(\theta)=\frac{T^{+}(\theta)-T^{-}(\theta)}{T^{+}(\theta)+T^{-}(\theta)} .
$$

Substituting for the neutron transmissions for each spin state, and assuming $\sigma_{\mathrm{FC}} \ll \sigma_{T}$, gives

$$
\epsilon(\theta)=n \widetilde{t_{10}}(s) \widetilde{t_{20}}(I) \sigma_{\mathrm{FC}}(\theta),
$$

which relates the asymmetry to the fivefold correlation term.

It is customary to remove the angular dependence of $\sigma_{\mathrm{FC}}$ and quote a bound on a new spin-correlation coefficient $A_{5}$ defined by

$$
A_{5}=\frac{1}{\sin 2 \theta} \frac{\sigma_{\mathrm{FC}}(\theta)}{\sigma_{00}} .
$$

$A_{5}$ is related to the neutron asymmetry via

$$
A_{5}=\frac{a_{2}(1+\phi)}{\widetilde{t_{10}}(s) \widetilde{t}_{20}(I) n \sigma_{00}},
$$

where $a_{2}$ is the magnitude of the $\sin 2 \theta$ component in $\epsilon(\theta)$ [see Eqs. (24)-(25) later] and the factor $\phi$ accounts for the small number of $\gamma$ 's detected and counted as neutrons (Sec. IV). The spin-correlation coefficient $A_{5}$ is a direct measure of a parity-conserving, time-reversal violating quantity.

The deformation effect cross section, $\sigma_{02}$, is a measure of the nonspherical nuclear mass distribution. It is independent of the neutron polarization, depending only on the alignment of the target. The explicit expression for the deformation effect term in the cross section for our geometry is

$$
\begin{aligned}
\sigma_{02}= & 4 \pi \chi^{2} \frac{\sqrt{5}}{\hat{s}^{2} \hat{I}} P_{2}(\cos \theta) \sum_{J \ell j \ell^{\prime} j^{\prime}}(-1)^{s-\ell-j^{\prime}} \\
& \times(2 J+1) \hat{\ell} \hat{J}^{\prime} \hat{J}^{\prime}\left\langle\ell 200 \mid \ell^{\prime} 0\right\rangle W\left(J j I 2 ; I j^{\prime}\right) \\
& \times W\left(\ell j \ell^{\prime} j^{\prime} ; s 2\right) \operatorname{Im}\left\{T_{\ell^{\prime} j^{\prime} \ell j}^{J}\right\} .
\end{aligned}
$$

The $P_{2}(\cos \theta)$ angular dependence is evident.

Holmium is spin $I=\frac{7}{2}$ and, in principle, the cross section contains higher-order terms $(K=4,6)$. These terms are not important however, due to the small values of the tensor alignment terms $\widetilde{t}_{40}(I)$ and $\widetilde{t}_{60}(I)$. Studying the deformation term provides information on the degree and direction of the alignment of the target.

\section{EXPERIMENTAL APPARATUS AND PROCEDURES}

The experimental setup is shown in Fig. 1. A vertically $( \pm \hat{\mathbf{y}})$ polarized neutron beam is produced with momentum $\hat{\mathbf{k}}$, directed along $\hat{\mathbf{z}}$, and is transmitted through a ${ }^{165}$ Ho target 


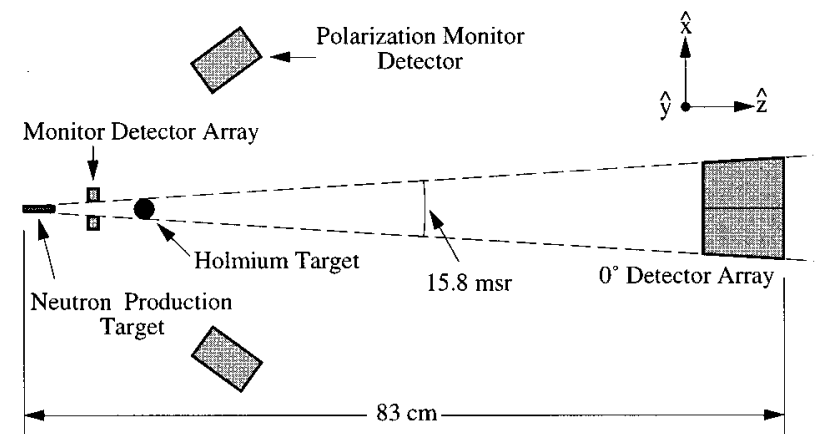

FIG. 1. The experimental setup for the fivefold correlation measurement. Vertically $( \pm \hat{\mathbf{y}})$ polarized neutrons with momentum $\hat{\mathbf{k}}$, directed along $\hat{\mathbf{z}}$, are transmitted through a nuclear-spin aligned holmium target and detected at $0^{\circ}$. The dashed lines depict the solid angle subtended by the neutron detectors. All components and distances are drawn to scale.

with nuclear-spin alignment in the $\hat{\mathbf{x}}-\hat{\mathbf{z}}$ plane. The transmitted flux is detected at $0^{\circ}$. The incident flux is monitored between the source and target, and the neutron polarization is determined from a pair of left/right detectors.

\section{A. Neutron beam}

The polarized neutron beam is produced via the ${ }^{2} \mathrm{H}(\vec{d}, \vec{n}){ }^{3} \mathrm{He}$ reaction, using a polarized deuteron beam from the TUNL atomic beam polarized ion source. The neutron spin is reversed by flipping the direction of the deuteron spin in the polarized ion source. The deuteron beam is accelerated to $4.9 \mathrm{MeV}$ using a Tandem Van de Graaff accelerator and collimated to form a $0.625 \mathrm{~cm}$ diameter beam. This beam enters a deuterium gas cell $(3.81 \mathrm{~cm}$ long, $0.851 \mathrm{~cm}$ diameter, filled to 8 bars) through a $15.2 \mu \mathrm{m}$ Havar foil and is stopped by a $0.51 \mathrm{~mm}$ gold foil surrounding the deuterium gas. The density of the gas is increased by cooling the cell using a thermal connection to a liquid-nitrogen bath. The temperature of the cell is monitored and regulated at $168 \pm 0.5 \mathrm{~K}$.

With a gas thickness of $\rho t=8.84 \mathrm{mg} / \mathrm{cm}^{2}$, a $4.9 \mathrm{MeV}$ deuteron beam produces a neutron beam with an average energy of $5.9 \mathrm{MeV}$. The energy loss of the deuteron beam in the gas cell produces an energy spread of $2.6 \mathrm{MeV}$. The neutron beam energy is verified by locating resonances in oxygen near $6 \mathrm{MeV}$.

Two liquid scintillator (NE-213) neutron detectors are symmetrically located at $\pm 36^{\circ}$ with respect to the beam direction to monitor the neutron polarization. The detectors are mounted in the horizontal $x-z$ plane and face the center of the deuterium gas cell. The neutron polarization is measured using the left-right analyzing power of the ${ }^{2} \mathrm{H}(d, n)^{3} \mathrm{He}$ reaction. The average asymmetry for the two spin states, formed in a manner to remove the unknown detector efficiencies, is given by

$$
\epsilon_{L R}=\frac{\sqrt{\left(N_{L}^{+} / N_{L}^{-}\right)\left(N_{R}^{-} / N_{R}^{+}\right)}-1}{\sqrt{\left(N_{L}^{+} / N_{L}^{-}\right)\left(N_{R}^{-} / N_{R}^{+}\right)}+1},
$$

where $L$ and $R$ denote the left and right detectors and \pm denote the neutron spin state. The left-right asymmetry was measured to be $\epsilon_{L R}=0.048 \pm 0.001$ during the time-reversal measurement. After combining this value with the known parameters of the ${ }^{2} \mathrm{H}(\vec{d}, n)^{3} \mathrm{He}$ reaction [22], a neutron polarization of $\widetilde{t_{10}}(s)=0.67 \pm 0.05$ is determined.

The neutron yield depends on the tensor polarization $P_{z z}$ of the deuteron beam via $I^{ \pm}=I_{0}\left(1-\frac{1}{4} A_{z z} P_{z z}^{ \pm}\right)$where $A_{z z}$ is the tensor analyzing power of the ${ }^{2} \mathrm{H}(\vec{d}, n)^{3} \mathrm{He}$ reaction. For our source, the difference $\left|P_{z z}^{+}-P_{z z}^{-}\right| \sim 0.01$, giving a constant term in the asymmetry (independent of $\theta$ ) of $5 \times 10^{-4}$.

\section{B. Collimation and detection}

Detectors are positioned such that unscattered neutrons must pass through the holmium target to be detected. The solid angle is fixed by the entrance window of the deuterium gas cell and the edges of the holmium target. The $0^{\circ}$ detectors are placed $70 \mathrm{~cm}$ behind the target and subtend a solid angle of $15.8 \mathrm{msr}$.

A four-detector array of Pilot-U scintillation material monitors a halo of the beam emerging from the gas cell. Each scintillator is $1.26 \mathrm{~cm}$ thick and is connected to a 2.86 $\mathrm{cm}$ photomultiplier tube through a polished lucite light guide. The transmitted beam is detected in a second Pilot-U scintillator array. Each scintillator consists of a $6.35 \times 6.35 \times 10.12 \mathrm{~cm}$ long rectangular piece of Pilot-U connected to a $5.08 \mathrm{~cm}$ photomultiplier tube via a polished lucite light guide.

Due to the high counting rates in the monitor and $0^{\circ}$ detector arrays, a transistorized voltage divider circuit is used to maintain linearity in the high gain stages of the photomultiplier tubes. The voltage divider circuits are assembled on a printed circuit board and are attached directly to the photomultiplier tube bases.

Monte Carlo calculations of the inscattering from surrounding material and small-angle scattering within the holmium target are performed using the program MCNP [23]. These calculations indicate that less than $1 \%$ of the counts in the main detector arise from small-angle scattering from the holmium target and less than $1 \%$ of the counts arise from scattering from surrounding material. No corrections are made to the data for inscattering.

\section{Target}

Holmium is chosen as the target nucleus because it is monoisotopic $\left({ }^{165} \mathrm{Ho}\right)$ and single crystals have a large internal hyperfine field. The internal field orients the nuclei when the thermal energy becomes comparable to or less than the hyperfine energy splitting.

The crystal structure of metallic holmium is hexagonal close packed. Below $20 \mathrm{~K}$, holmium undergoes a magneticordering transition to a ferromagnetic state [24]. In this state, the magnetic moments are directed $10^{\circ}$ out of the $a-b$ basal plane and are parallel within a given plane. In successive planes, the moments are rotated $30^{\circ}$ about the $c$ axis, forming a cylindrically symmetric spiral structure about the $c$ axis. In the absence of an external magnetic field, nuclear alignment occurs when the sample is cooled below $10 \mathrm{~K}$.

The alignment of the nucleus along the axis of its magnetic moment is given by [25] 


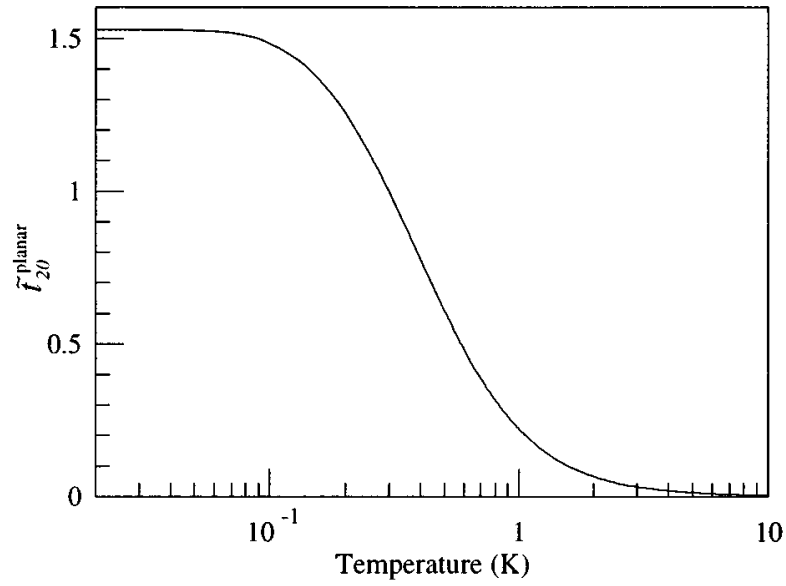

FIG. 2. The nuclear-spin alignment of holmium as a function of temperature. The planar alignment is related to the polarization tensor along the symmetry axis via Eq. (14).

$$
\tilde{t}_{20}^{\text {planar }}=6 \sqrt{5} \hat{I} \sqrt{\frac{(2 I-2) !}{(2 I+3) !}}\left[\sum_{m} m^{2} a_{m}-\frac{1}{3}(I+1)\right] \text {, }
$$

where $I$ is the nuclear spin and $a_{m}$ is given by the Boltzmann probability distribution. The symmetry axis of the alignment is directed along the $c$ axis of the crystal and only the $c$ axis components of the magnetic moments contribute to the alignment. The polarization tensor along the symmetry axis is related to the planar alignment tensor via

$$
\widetilde{t_{20}}=P_{2}\left(\cos 80^{\circ}\right) \widetilde{t}_{20}^{\text {planar }},
$$

where $80^{\circ}$ corresponds to the angle between the magnetic moments and the $c$ axis of the crystal. The value of $\widetilde{t}_{20}^{\text {planar }}$ for holmium as a function of temperature is shown in Fig. 2 with the maximum value of the polarization tensor being

$$
\widetilde{t}_{20}=\sqrt{\frac{7}{3}} P_{2}\left(\cos 80^{\circ}\right)=-0.695 \text {. }
$$

The single-crystal sample used in this experiment was grown by Ames Laboratory ${ }^{1}$ and machined into a cylindrical shape with the $c$ axis perpendicular to the cylinder axis. The sample is mounted with the cylinder axis along the vertical $y$ axis, placing the alignment axis in the $x-z$ plane. The 101.6 $\mathrm{g}$ sample is $99.8 \%$ holmium. The cylinder is $2.29 \mathrm{~cm}$ diameter and $2.8 \mathrm{~cm}$ in height.

The holmium sample is cooled to $160 \mathrm{mK}$ by a ${ }^{3} \mathrm{He}-{ }^{4} \mathrm{He}$ dilution refrigerator. A central shaft extends from room temperature to the sample holder to allow the target to be rotated at this temperature. The design and construction of the refrigerator and rotation apparatus is discussed by Koster [26].

The sample is mounted in a copper cylinder using a pressfit connection. This holder is connected to the refrigerator through three copper braids. Each braid consists of 110

\footnotetext{
${ }^{1}$ Materials Preparation Center at Iowa State University.
}

strands of $0.016 \mathrm{~cm}$ copper wire extending $15 \mathrm{~cm}$. The braids allow the target to be rotated a full $360^{\circ}$ about its cylinder axis.

The temperature of the target is measured using resistance thermometry and confirmed using measurements of the deformation effect cross section (Sec. IV). To avoid temperature changes due to rotation, a second thermometer is used in combination with a $500 \Omega$ heater to stabilize the temperature to $\pm 2 \mathrm{mK}$ during collection of the data.

The target alignment is calculated using the temperature of the sample and the known temperature dependence of the alignment. The uncertainty in the temperature arises from both the fluctuations in temperature and the calibration of the resistance thermometer. This thermometer is calibrated against a germanium thermometer whose calibration is $3 \%$ at this temperature, resulting in an uncertainty of $\sim 5 \%$ in the thermometer calibration. This yields an uncertainty in the sample temperature of $\pm 8 \mathrm{mK}$. Combining this value with the temperature and known values of the energy eigenvalues of the magnetic dipole and electric quadrupole interactions [27], a target alignment of $\widetilde{t}_{20}^{\text {planar }}=1.36 \pm 0.07$ is determined. This is approximately $90 \%$ of the maximum value.

\section{Electronics}

The data are collected using standard NIM and CAMAC electronics. Counts from the neutron detectors are stored for each polarized ion source spin state and target alignment angle.

The $0^{\circ}$ and monitor detector anode signals trigger 300 $\mathrm{MHz}$ discriminators, and the events above threshold are counted with a 32 channel, $225 \mathrm{MHz}$ scaler. With $2.0 \mu \mathrm{A}$ of beam, count rates of 4.4 and $14.1 \mathrm{MHz}$ are observed for the $0^{\circ}$ and monitor detector arrays, respectively.

The neutron spin is toggled every $100 \mathrm{~ms}$ in the eight-step sequence +--+-++- . Two locally designed NIM modules control the spin-flip sequence and the data acquisition system. An external clock input drives the spin-state controller (SSC) which is used to (a) toggle the rf cavities in the polarized ion source in order to reverse the spin of the deuteron beam and (b) to route the data according to the neutron spin state.

A second module controls the SSC and vetoes the data flow during the time the spins are being flipped. The veto signal is $7 \mathrm{~ms}$ in length, beginning $2 \mathrm{~ms}$ before the spin flip. An additional pulse is generated $1 \mathrm{~ms}$ before the spin flip and is used to initiate the scaler read.

A separate $50 \mathrm{MHz}$ oscillator is used as a pulser to monitor the time spent in each neutron spin state. The pulses are counted with the same 32 channel scaler and routed in the same manner as the data. This information is used to verify equal time is spent in each spin state.

The target rotation occurs at the end of each run of either 256 or 1024 eight-step sequences. The rotation is controlled by a computer connected to a Texas Instruments Model 525 Programmable Controller. Either a $22.5^{\circ}$ or $90^{\circ}$ rotation is performed after each run and takes approximately $20 \mathrm{~s}$ to complete. Data collection is inhibited until the angle has been reached. A data set consists of a $360^{\circ}$ rotation of the target and takes about $1 \mathrm{~h}$ to complete. 


\section{E. Procedures}

The experiment begins with a $4 \mathrm{~h}$ measurement of the deformation effect at $9.4 \mathrm{MeV}$ to verify the target alignment. Next, the energy of the neutron beam is lowered to $5.9 \mathrm{MeV}$, where the deformation effect is small and sensitivity to the time-reversal signal is a maximum [14]. Data are collected for seven days, stopping every $24 \mathrm{~h}$ to fill the cryostat with liquid nitrogen and liquid helium.

The alignment axis of the target is initially varied every 256 eight-step sequences in the angular sequence $-180^{\circ} \rightarrow+180^{\circ} \rightarrow-180^{\circ}$ in $22.5^{\circ}$ steps. This sequence provides information on 15 angular components in the asymmetry [see Eq. (24) later] which is useful in constraining systematics. The alignment angle is then varied every 1024 eight-step sequences in the angular sequence $-135^{\circ} \rightarrow+135^{\circ} \rightarrow-135^{\circ}$ in $90^{\circ}$ steps. This sequence provides maximum sensitivity to the $\sin 2 \theta$ component in the asymmetry.

At the end, a second deformation effect measurement is performed to verify the alignment obtained from thermometry and to verify the operation of the rotation apparatus. Supplementary measurements are also performed, as discussed in the next section.

\section{SUPPLEMENTAL MEASUREMENTS}

Analysis of the time-reversal data requires knowledge of various parameters not measurable during the experiment. These include multiple-scattering events within the detectors, background $\gamma$-ray count rates, and estimates of $T$-conserving interactions that could mimic a $\sin 2 \theta$ angular dependence. This section serves to present these measurements.

\section{A. Multiple-scattering events}

The segmentation of the neutron detectors gives rise to multiple-scattering events within two or more detectors. These events are counted in multiple detectors, thereby artificially increasing the neutron count rate. The multiplescattering events - or cross-talk events-occur when a neutron or $\gamma$-ray is scattered within one scintillator and then scatters within another. The large neutron flux prohibits the use of coincidence rejection techniques, but the cross talk can be measured at lower neutron fluxes and a correction factor determined for each detector pair.

The total number of counts in detector number $i, N^{i}$, contains the actual yield $N_{0}^{i}$, plus an additional term due to the cross talk. Defining $\chi(a, b)$ as the cross-talk ratio for a detector pair, the neutron yield for detector number one, for example, is

$$
N^{1}=[1+\chi(1,2)+\chi(1,3)+\chi(1,4)] N_{0}^{1} .
$$

Twofold coincidence measurements contain both the crosstalk and accidental rate. We define $\eta(a, b)$ to be the ratio of the number of twofold coincidences between detectors $a$ and $b$ to the total count rate in the two detectors. This ratio is related to the cross talk via

$$
\eta(a, b)=\frac{\chi(a, b)\left(N_{0}^{a}+N_{0}^{b}\right)}{\left(N^{a}+N^{b}\right)}+\frac{k I^{2}}{\left(N^{a}+N^{b}\right)},
$$

TABLE I. Measured cross-talk ratio between detector pairs. The numbering scheme is (1) upper left, (2) upper right, (3) lower right, and (4) lower left with respect to the beam direction.

\begin{tabular}{lcc}
\hline \hline Detector pair & $0^{\circ}(\%)$ & Monitor $(\%)$ \\
\hline$\chi(1,2)$ & $0.678 \pm 0.006$ & $0.010 \pm 0.002$ \\
$\chi(1,3)$ & $0.183 \pm 0.004$ & $0.006 \pm 0.002$ \\
$\chi(1,4)$ & $0.759 \pm 0.007$ & $0.014 \pm 0.002$ \\
$\chi(2,3)$ & $0.654 \pm 0.006$ & $0.015 \pm 0.002$ \\
$\chi(2,4)$ & $0.161 \pm 0.004$ & $0.007 \pm 0.002$ \\
$\chi(3,4)$ & $0.727 \pm 0.007$ & $0.034 \pm 0.002$ \\
\hline \hline
\end{tabular}

where the second term denotes the accidental rate. The accidental rate scales quadratically with beam current $I$, with $k$ denoting the proportionality constant. We take $N^{a} \approx N_{0}^{a}$ and $N^{a} \propto I$ yielding

$$
\eta(a, b)=\chi(a, b)+k^{\prime} I
$$

Thus, by measuring the ratio $\eta(a, b)$ at two values of $I$, $\chi(a, b)$ can be extracted.

The cross-talk measurements are performed for both the $0^{\circ}$ and monitor detectors at $5.9 \mathrm{MeV}$ using two different beam currents: $I=1.25$ and $0.125 \mu \mathrm{A}$. Two measurements are performed: total count rates for each detector and twofold coincidences between detector pairs. These measurements are performed for both detector arrays and for each beam current configuration. The three- and fourfold coincidence rates are at least two orders of magnitude smaller than the twofold rate and do not contribute.

Using measured twofold coincidence values, the cross talk for each detector pair is determined and is summarized in Table I. The results indicate cross-talk effects are $<1 \%$.

\section{B. Ratio of neutrons to $\gamma$ rays}

The use of plastic scintillators prohibits pulse shape discrimination between neutrons and $\gamma$ rays. The ratio of neutrons to $\gamma$ rays is measured for the $0^{\circ}$ detector array using time-of-flight techniques. A typical time-of-flight spectrum is shown in Fig. 3. Each channel corresponds to $0.17 \mathrm{~ns}$.

The ratio of neutrons to $\gamma$ rays in an individual detector is determined by dividing the number of counts in the $\gamma$-ray peak by the number of counts in the neutron peak. Since the discriminators are set identically for all four detectors, the individual ratios are averaged to determine a single normalization factor. This factor is measured to be $\phi=6.38 \pm 0.01 \%$.

Measurements of the time-of-flight spectrum for the monitor detectors are not possible due to the short distance separating the detectors from the neutron production cell. The background of the $\gamma$ rays is expected to be much smaller than observed in the $0^{\circ}$ detectors due to the reduced thickness of the scintillation material. This ratio is estimated to be less than $1 \%$ and no corrections are made in the analysis of the time-reversal data.

\section{Deformation effect}

Measurements of the deformation effect cross section are performed to both verify the tensor alignment of the hol- 


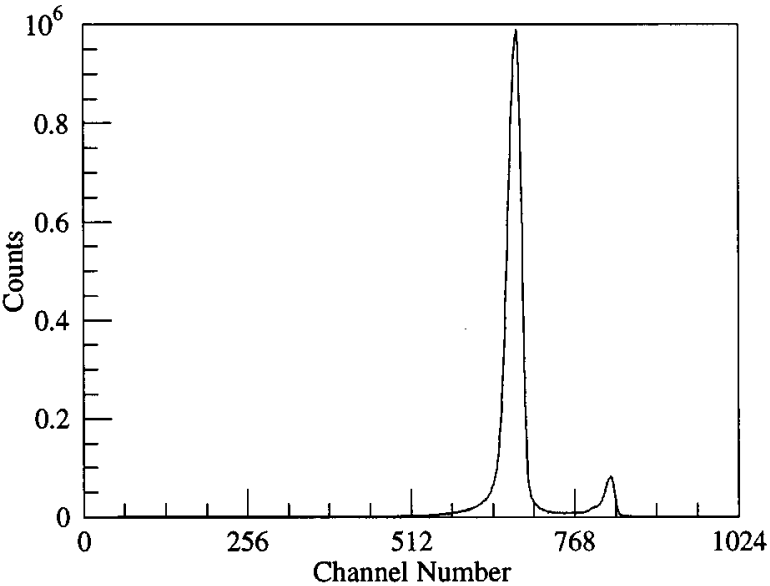

FIG. 3. Typical time-of-flight spectrum for a $0^{\circ}$ detector at 5.9 $\mathrm{MeV}$ neutron energy. Time increases from right to left. The large peak corresponds to neutrons and the small peak corresponds to $\gamma$ rays.

mium target obtained from thermometry and to confirm the direction of the alignment axis of the holmium crystal. These measurements are performed immediately before and after the time-reversal data are taken. Measurements of the deformation effect cross section at four additional energies are also performed during the initial testing stages of the experiment.

Deformation effect cross-section measurements are made by transmitting unpolarized neutrons through an aligned ${ }^{165} \mathrm{Ho}$ target. The $0^{\circ}$ neutron yield is proportional to the total cross section, which for an unpolarized neutron beam, consists of the unpolarized and deformation effect partial cross sections:

$$
\sigma_{T}=\sigma_{00}+\widetilde{t}_{20}(I) \sigma_{02}
$$

The deformation effect term exhibits a $P_{2}(\cos \theta)$ angular signature. By varying the alignment angle with respect to the beam direction, this angular signature is used to extract the product $\widetilde{t}_{20}(I) \sigma_{02}$ from the total cross section. Comparison with previous deformation effect measurements provides an independent verification of the magnitude of the tensor alignment of the holmium target.

At each of the six energies at which $\sigma_{02}$ is measured, four to six hours of data are taken with the angle $\theta$ between the target alignment axis and neutron momentum varied every four minutes in the angular sequence $-180^{\circ} \rightarrow$ $+180^{\circ} \rightarrow-180^{\circ}$ in $22.5^{\circ}$ steps. The neutron yield as a function of run number (or angular sequence) is used to extract the deformation effect term in the total cross section. Data from a measurement of the neutron yield at $9.4 \mathrm{MeV}$ are shown in Fig. 4. The oscillation is due to the deformation effect and the slow linear drift is due to gain drifts in the photomultiplier tubes.

The logarithm of the normalized neutron yield as a function of angle- $\ln \left(N(\theta) / N_{0}\right)$-is fit to the form $C+A P_{2}(\cos \theta)$ to extract the magnitude of the deformation effect term. As with the fivefold correlation term discussed earlier, the angular dependence is removed $\left[\sigma_{2}=\right.$ $\left.\sigma_{02} / P_{2}(\cos \theta)\right]$, yielding a quantity that can be directly com-

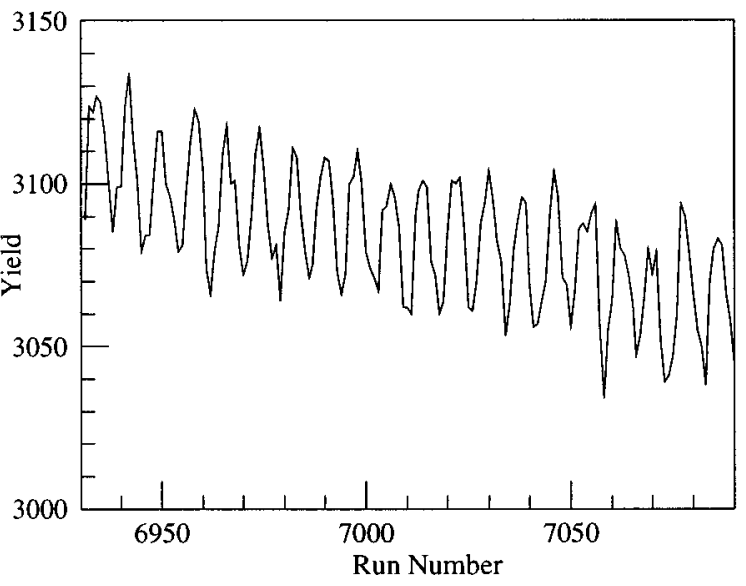

FIG. 4. The transmission yield for 9.4 MeV neutrons. Each run number corresponds to four minutes of data taken in the angular sequence $-180^{\circ} \rightarrow+180^{\circ} \rightarrow-180^{\circ}$ in $22.5^{\circ}$ steps. The oscillation arises from the deformation effect.

pared to previous measurements. The angular coefficient $A$ from the fit is related to the deformation effect cross section via

$$
\sigma_{2}=\frac{A}{n \widetilde{t_{20}}(I)},
$$

where $n=0.065 \mathrm{at} / \mathrm{b}$ is the target thickness and $\widetilde{t}_{20}(I)$ is the tensor alignment of the holmium target.

Measurements of the deformation effect cross section are presented in Table II and shown in Fig. 5. The $1.93 \mathrm{MeV}$ measurement is made using the ${ }^{3} \mathrm{H}(\vec{p}, \vec{n}){ }^{3} \mathrm{He}$ neutron production reaction. The remaining measurements are performed using the ${ }^{2} \mathrm{H}(\vec{d}, \vec{n})^{3} \mathrm{He}$ reaction. The new values are consistent with previous measurements, thus confirming the target alignment obtained from thermometry.

The phase of the angular variation of the cross section also determines the direction of the alignment axis of the crystal. The alignment axis was previously located using $\mathrm{x}$-ray-diffraction techniques and the deformation effect measurements are used as independent verification. The phase is extracted by performing a nonlinear fit to the function $C+A P_{2}(\cos (\theta+\delta))$. A value of $\delta=1.9^{\circ} \pm 2.2^{\circ}$ is extracted for the phase of the oscillation. This phase is consistent with zero and taken to be zero in subsequent analyses.

TABLE II. Measured values of the deformation effect cross section and the tensor alignment of the holmium target obtained from thermometry during these measurements.

\begin{tabular}{ccc}
\hline \hline Energy $(\mathrm{MeV})$ & $\tilde{t}_{20}(I)$ & $\sigma_{2}(\mathrm{mb})$ \\
\hline 1.93 & -0.59 & $-246 \pm 41$ \\
5.86 & -0.62 & $5 \pm 3$ \\
6.57 & -0.59 & $-3 \pm 8$ \\
8.41 & -0.59 & $-174 \pm 23$ \\
9.37 & -0.62 & $-237 \pm 7$ \\
10.5 & -0.59 & $-338 \pm 44$ \\
\hline \hline
\end{tabular}




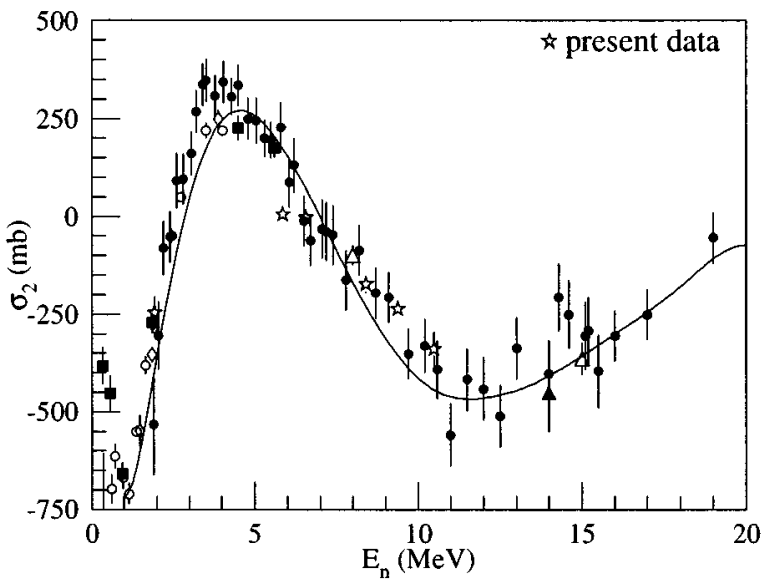

FIG. 5. The deformation effect cross section as a function of neutron energy. The solid curve corresponds to an optical model fit given by Koster et al. [28] with the other data referenced therein.

In order to reduce systematic effects that might arise from the deformation effect term, the time-reversal measurement is performed at $5.9 \mathrm{MeV}$ where the deformation effect cross section is small. These effects and others are discussed further in the next section.

\section{FALSE ASYMMETRIES}

$T$-conserving terms that can mimic a $\sin 2 \theta$ angular dependence in the normalized asymmetry must be considered. These false asymmetry terms can arise from either sequential interactions or from systematic effects. In this section we show that false asymmetries are below the sensitivity of the present measurement. Table III is a brief summary of the effects considered and/or measured.

\section{A. Sequential interactions}

The sequential interaction terms are present independent of any systematic considerations. Stodolsky [29] identified these terms, and showed how they can be removed by analyzing the spin of the neutron beam after it passes through the target. Kabir [30] expanded this discussion to include all

TABLE III. False asymmetry terms and upper bounds on their contributions to a $\sin 2 \theta$ term in the asymmetry.

\begin{tabular}{lcr}
\hline \hline Source & $\sin 2 \theta ?$ & Asymmetry \\
\hline Tensor polarization difference & No & $5 \times 10^{-4}$ \\
Mott-Schwinger+deformation & No & $10^{-5}$ \\
Sequential interactions & Yes & $<10^{-9}$ \\
Neutron spin misalignment & Yes & $<10^{-6}$ \\
Rotation axis misalignment & No & $<5 \times 10^{-8}$ \\
Detector misalignment & No & $<10^{-8}$ \\
Target $c$-axis misalignment & Yes & $<7 \times 10^{-7}$ \\
Target thickness variation & No & $<10^{-6}$ \\
Gas cell beam heating & Yes & $<3 \times 10^{-7}$ \\
$(\gamma, \mathrm{n})$ reactions & No & $<10^{-7}$ \\
Spin-spin interactions & No & $<10^{-7}$ \\
\hline \hline
\end{tabular}

possible combinations of reactions that can mimic a $T$-violating signal and how such an analyzer removes these effects.

Analyzing the exiting neutron polarization, although unambiguous in determining the $T$-violating asymmetry, is a very inefficient procedure because it requires a second scattering process, effectively reducing the number of neutrons detected by $\sim 10^{4}$. Measuring an asymmetry at the $10^{-6}$ level in this way is therefore completely impractical. For fivefold correlation measurements however, the two $T$-conserving terms, $\hat{\mathbf{s}} \cdot \hat{\mathbf{k}}$ and $(\hat{\mathbf{s}} \cdot \hat{\mathbf{I}})(\hat{\mathbf{I}} \cdot \hat{\mathbf{k}})$, that can combine to generate the fivefold angular signature are both parity violating [31] and are therefore expected to be small.

The $(\hat{\mathbf{s}} \cdot \hat{\mathbf{k}})$ term was investigated by Soderstrum et al. [32] and found to be $(-2.4 \pm 2.6) \times 10^{-4}$. The $(\hat{\mathbf{s}} \cdot \hat{\mathbf{I}})(\hat{\mathbf{I}} \cdot \hat{\mathbf{k}})$ term is investigated in the present experiment (see below) and found to be $(-1.0 \pm 1.2) \times 10^{-5}$. When combined, effects from the sequential interaction of these terms must be $\lesssim 10^{-9}$, well below the limits of the present measurement. No other sequential interaction terms can combine to generate a $\sin 2 \theta$ dependence [31].

\section{B. Systematic effects}

Systematic effects that could lead to a false asymmetry include detector and target misalignments and finite-size effects. Although most of these effects cannot generate the $\sin 2 \theta$ angular signature of the FC, upper bounds on the magnitude of these interactions must be estimated before excluding them in the analysis.

\section{Misalignments}

Only one misalignment term can directly contribute to a $\sin 2 \theta$ angular dependence. This is the partial cross section $\sigma_{12}(\Lambda=1)$ which depends on the parity-violating $(\hat{\mathbf{s}} \cdot \hat{\mathbf{I}})(\hat{\mathbf{I}} \cdot \hat{\mathbf{k}})$ interaction. The angular dependence arises when the neutron spin has a component directed along the $x$ axis, perpendicular to the momentum of the beam. This term is expected to be negligible because it is parity violating and must couple with the deformation effect to present an effect. However, we have attempted to set a bound on this term experimentally by placing the neutron spin along the $x$ axis to maximize the effect. The $\sin 2 \theta$ contribution to the asymmetry was measured to be $(-1.0 \pm 1.2) \times 10^{-5}$, consistent with zero. In the time-reversal measurement the neutron spin is directed along the vertical $y$ axis or perpendicular to the horizontal $x-z$ plane, so contributions from this term are suppressed by at least an order of magnitude. We therefore conclude that this term is not present to the level studied in the PC TRNI measurement.

Other false asymmetries due to misalignments give a $\sin 2 \theta$ dependence only indirectly. First consider a misalignment of the rotation axis of the target. This can produce a reduced target thickness because some of the neutron beam reaching the detectors does not pass through the holmium target. The target is aligned optically and is centered to within $0.25 \mathrm{~mm}$ at room temperature. A $1 \mathrm{~mm}$ displacement would reduce the target thickness by $5 \times 10^{-3}$. To simulate a time-reversal violating signature, this effect must couple with both an angle-dependent interaction such as the deformation effect $\left(\sim 10^{-3}\right)$ and an analyzing power effect 
$\left(\sim 10^{-2}\right)$ in order to produce a spin-dependent angular signature. This effect is thus $\leqslant 5 \times 10^{-8}$ for a $1 \mathrm{~mm}$ displacement, and is expected to be much smaller because of the symmetric design of the mounting apparatus of the target.

Variations in the target thickness can also give rise to angle-dependent effects. For example, if the cross sectional area of the target is oval in shape or the target moves from left to right, this could, when coupled with a holmium analyzing power effect, lead to an angular dependence in the asymmetry. The thickness of the target is circular to within $0.1 \mathrm{~mm}$, leading to a maximum transmission asymmetry $\sim 10^{-4}$. Similarly, the left-right movement of the target is less than $0.5 \mathrm{~mm}$, with a maximum transmission asymmetry of $\sim 10^{-4}$. The $n+{ }^{165}$ Ho analyzing power reaction further reduces this effect by $\sim 10^{-2}$. The effect is thus $\lesssim 10^{-6}$. In fact, it is only a problem if both the $0^{\circ}$ detector is misaligned and the angular signature arising from the variation in target thickness has a $\sin 2 \theta$ angular dependence, neither of which is evident in the data.

A misalignment of either the zero degree or monitor detector array leads to a constant offset in the asymmetry due to the analyzing power of the ${ }^{2} \mathrm{H}(\vec{d}, \vec{n})^{3} \mathrm{He}$ reaction. Alignment of the detectors is performed using an optical transit and the detectors are centered to within $2 \mathrm{~mm}$, yielding a maximum misalignment angle of $0.2^{\circ}$. The false asymmetry arising from such a misalignment is calculated to be $\lesssim 10^{-5}$. This term can only contribute when combined with an angular-dependent interaction such as the deformation effect $\left(\sim 10^{-3}\right)$. These effects are thus $\lesssim 10^{-8}$ and are therefore negligible.

Large analyzing powers from small-angle neutron scattering within the holmium sample are possible via MottSchwinger scattering. These effects have been measured for neutron scattering in lead [33], having values as large as $A_{y}\left(1^{\circ}\right) \simeq 1.0$. These effects are symmetric about $0^{\circ}$ and the large size of the detectors will tend to average out their contributions. The outer edges of the $0^{\circ}$ detector are at $\pm 5^{\circ}$ with respect to the holmium target and the analyzing power at this angle is $\simeq 0.2$. An effect from this term would arise from either a misalignment of the $0^{\circ}$ detector, causing the average analyzing power on the two sides of the detector to be unequal, or a nonuniform neutron beam polarization, yielding a preferential scattering of neutrons from one side of the target. For a $0.2^{\circ}$ misalignment of the $0^{\circ}$ detector array, a contribution to the asymmetry is $\sim 10^{-2}$. For a $5 \%$ greater neutron polarization on one side of the target, the contribution to the asymmetry is also $\sim 10^{-2}$. In fact, a $\cos 2 \theta$ variation in the asymmetry arising from the deformation effect $\left(\sim 10^{-3}\right)$ coupled with an analyzing power reaction effect is present at the $\sim 10^{-5}$ level, consistent with these estimations.

A misalignment of the target's alignment axis can present a problem if the $\cos 2 \theta$ term is nonzero. The misalignment mixes the amplitudes of the $\sin 2 \theta$ and $\cos 2 \theta$ terms in the asymmetry; $A(\sin \delta \sin 2 \theta+\cos \delta \cos 2 \theta)$, where $\delta$ is the misalignment angle of the $c$ axis. This cannot be large because the deformation effect measurements confirm that the alignment axis is known to within $\delta=4.1^{\circ}$. A $4.1^{\circ}$ misalignment combined with a $\cos 2 \theta$ term of $(8.5 \pm 1.5) \times 10^{-6}$ gives a $\sin 2 \theta$ component of at most $7 \times 10^{-7}$. Therefore, the $\cos 2 \theta$ term does not lead to an observable $\sin 2 \theta$ signal.

\section{Beam-related effects}

When the deuteron beam is stopped in the gas cell, it deposits up to $6 \mathrm{~W}$ of heat. This produces density variations in the gas, which in turn, cause variations in the neutron flux. This heat is removed by the liquid-nitrogen bath, but the system has a $1 \mathrm{~h}$ thermal time constant. As a result, density changes are time dependent. Since the angle sequence is correlated in time, there is the potential for deuteron beam intensity variations to give neutron yield variations which are correlated with angle. In earlier tests when the gas cell was not temperature stabilized, we saw $\sin 2 \theta$ variations in the asymmetries due to beam heating effects. To eliminate these, we temperature stabilized the gas cell to within $0.5 \mathrm{~K}$ reducing the temperature variations by two orders of magnitude. Normalization to the monitor further reduces these effects by an order of magnitude to below $3 \times 10^{-7}$.

\section{Miscellaneous effects}

The neutrons produced in the giant dipole resonance $(\gamma, n)$ reactions can contribute to the asymmetry. Cross sections for these reactions are $\sim 20 \mathrm{mb}$ [34], two orders of magnitude smaller than the neutron total cross section. Further, the ratio of neutrons to $\gamma$ rays is measured to be $6.4 \%$. The $\gamma$ rays resulting from the stopped deuteron beam have energy of at most $\sim 1 \mathrm{MeV}$ and higher-energy $\gamma$ rays produced directly by the deuteron beam are not expected. The ratio of $\gamma$ rays alone suppresses contributions from $(\gamma, n)$ reactions by an order of magnitude and the small number of higher-energy $\gamma$ rays in the giant resonance region (10-20 $\mathrm{MeV}$ ) further suppresses this effect. An angle-dependent term such as the deformation effect $\left(\sim 10^{-3}\right)$ must couple with a spin-dependent analyzing power effect $\sim 10^{-1}$ to produce an angular signature. Effects from $(\gamma, n)$ reactions are thus $\leqslant 10^{-7}$.

Spin-spin cross sections are typically $\sim 10^{-3}$ in heavy nuclei, suppressed by a factor of $1 / A$. The polarization of the sample is $\widetilde{t}_{10}(I) \sim 10^{-4}$, based on the temperature and the residual magnetic field $(<1 \mathrm{G})$ near the sample. As a result we expect spin-spin effects to be $\sim 10^{-7}$ or less.

\section{DATA ANALYSIS}

Data are taken in two angular rotation sequences. The first data set is taken in the angular rotation sequence $-180^{\circ} \rightarrow+180^{\circ} \rightarrow-180^{\circ}$ in $22.5^{\circ}$ steps. At each angle, 256 eight-step neutron spin sequences are accumulated. The second data set is taken using the same eight-step neutron spin sequence but with an angular sequence of $-135^{\circ} \rightarrow+135^{\circ} \rightarrow-135^{\circ}$ in $90^{\circ}$ steps. At each angle, 1024 eight-step sequences are accumulated. This angle sequence maximizes sensitivity to the $\sin 2 \theta$ component of interest, but information on higher-order components is lost. The two data sets are analyzed as separate measurements and combined in the final step of the analysis. The first subset of data consists of 1568 runs of 256 sequences per run and the second subset contains 264 runs of 1024 sequences per run. Four rejection criteria are applied to the data to determine if an eight-step sequence is accepted or not: (a) equal amounts 


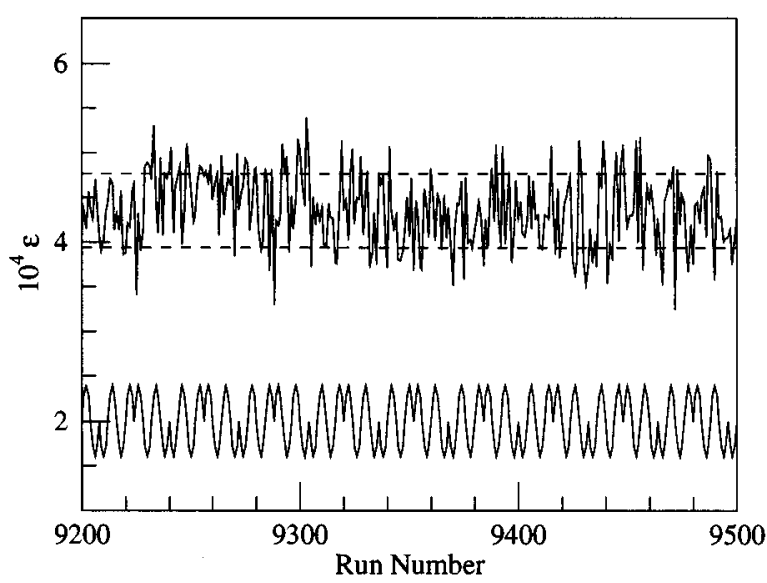

FIG. 6. Monitor normalized detector asymmetry $\epsilon$ as a function of run number for a 300 run subset of data. Each run corresponds to four minutes of data at a given angle, taken in the sequence $-180^{\circ} \rightarrow+180^{\circ} \rightarrow-180^{\circ}$ in $22.5^{\circ}$ steps. The dashed lines indicate the $\pm 1 \sigma$ errors on the data. The lower curve is a $\sin 2 \theta$ oscillation of arbitrary amplitude which would appear in the data if a $T$-violating signal were present. Based on fits to these and other data, we find the amplitude of the time-reversal violating $\sin 2 \theta$ term to be $(1.1 \pm 1.0) \times 10^{-6}$.

of time spent in each spin-state, (b) deviations of less than $20 \%$ in the average beam current in each spin state, (c) no rapid beam current fluctuations over the eight-step sequence, and (d) no rapid changes in the tensor polarization. This set of rejection criteria removes a total of $1.5 \%$ of the accumulated data.

A 300 run subset of data consisting of the average asymmetry for each run of 256 eight-step sequences is shown in Fig. 6 . The dashed lines indicate the $\pm 1 \sigma$ errors on the data. The lower curve is a sin $2 \theta$ oscillation of arbitrary amplitude which would appear in the data if a $T$-violating signal were present.

The neutron yields are corrected for both the dead time in the detectors and the cross talk between adjacent detectors. The dead time is dominated by the length of the $10 \mathrm{~ns}$ voltage pulse from the photomultiplier tubes, yielding for example, a $10 \%$ dead time at a count rate of $10 \mathrm{MHz}$ in a single detector. The dead-time corrections are applied individually to the detectors with typical corrections $\sim 0.5 \%$ for the $0^{\circ}$ detectors and $\sim 1 \%$ for the monitor detectors. Corrections for the cross talk between detector pairs are performed for both the $0^{\circ}$ and monitor detectors using the values presented in Table I.

The normalized asymmetry for an individual $0^{\circ}$ detector is calculated using the corrected yields:

$$
\epsilon_{i}=\frac{N_{i}^{+}\left(0^{\circ}\right) / N_{i}^{-}\left(0^{\circ}\right)-M}{N_{i}^{+}\left(0^{\circ}\right) / N_{i}^{-}\left(0^{\circ}\right)+M}
$$

where $N_{i}^{ \pm}\left(0^{\circ}\right)$ corresponds to the number of counts in a given $0^{\circ}$ detector and $M$ corresponds to the monitor normalization factor. To remove effects arising from gain drifts in the individual detectors, the monitor normalization factor is calculated using the ratio of the geometric means of the four monitor detectors:

$$
M=\sqrt[4]{\prod_{i=1}^{4} \frac{N_{i}^{+}(\mathrm{M})}{N_{i}^{-}(\mathrm{M})}}
$$

where $N_{i}^{ \pm}(\mathrm{M})$ corresponds to the number of counts in a given monitor detector. The geometric mean approximation is equal to the arithmetic mean for identical sample values and is slightly less than the arithmetic mean for numbers that are similar in size. For the present arrangement, the counts in the four monitor detectors are equal to within $10 \%$, allowing this approximation to be sufficient. The normalization to the geometric mean greatly reduces the nonstatistical fluctuations that would appear from detector efficiency changes and is therefore superior to the arithmetic mean.

The asymmetry for the $0^{\circ}$ detectors is formed by averaging the four individual asymmetries:

$$
\epsilon=\frac{1}{4} \sum_{i=1}^{4} \epsilon_{i}
$$

The error in $\epsilon$ however, cannot be calculated by combining the four individual errors $\Delta \epsilon_{i}$, since correlations exist due to the normalization to a single value of $M$. These correlations are taken into account in calculating the error in the asymmetry. The values of $\epsilon$ and $\Delta \epsilon$ for each spin sequence are then used in the extraction of the angular coefficients.

The magnitude of the angular coefficients in the asymmetry is extracted using a least-squares fit to the form

$$
\epsilon(x)=a_{0}+\sum_{k=1}^{7} a_{k} \sin (k \theta(x))+\sum_{k=1}^{8} b_{k} \cos (k \theta(x))
$$

for the $22.5^{\circ}$ rotation sequence and

$$
\boldsymbol{\epsilon}(x)=a_{0}+\sum_{k=1}^{2} a_{k} \sin (k \theta(x))+b_{1} \cos (\theta(x))
$$

for the $90^{\circ}$ rotation sequence. Here, $\theta(x)$ for the $22.5^{\circ}$ rotation sequence is $\theta(x)=\pi(x / 8-1)$ and for the $90^{\circ}$ rotation sequence is $\theta(x)=\pi(x / 2-3 / 4)$. The integer $x$ is given by $x=0,1, \ldots, m-1$ for a clockwise rotation and $x=m-1, m-2, \ldots, 0$ for a counterclockwise rotation. The number of angles in the sequence determines $m$, where for the $22.5^{\circ}$ rotation sequence $m=16$ and for the $90^{\circ}$ rotation sequence $m=4$. The number of trigonometric terms included in Eqs. (24) and (25) are determined using the angular rotation sequence with Nyquist's theorem. Higher-frequency components can exist, but will be aliased into the lowerfrequency terms.

The fits are performed using the Numerical Recipes leastsquares subroutine SVDFIT [35]. The angular position $\theta$ and the corresponding asymmetry $\epsilon \pm \Delta \epsilon$ for each eight-step sequence are used to extract the fitting coefficients $a_{k}$ and $b_{k}$ and their associated statistical errors and standard deviations. The fitting procedure was validated using randomly generated data sets consisting of Gaussian distributed noise on a constant background.

The results of the fits to the two sequences of experimental data are given in Table IV. The statistical error and standard deviation are in very close agreement. 
TABLE IV. The fitting coefficients of the least-squares fit to the two sets of time-reversal data. The first value corresponds to the fitting coefficient, the second to the statistical error, and the third to the standard deviation. The $T$ ratio is defined as the ratio of the fitting coefficient to the standard deviation.

\begin{tabular}{lcccc}
\hline \hline & & $22.5^{\circ}$ rotation sequence & & \\
Fitting Coefficient & & $T$ ratio & & Fitting Coefficient \\
\hline Constant & $43.32 \pm 0.11 \pm 0.11 \times 10^{-5}$ & 411.5 & $\cos \theta$ & $-0.47 \pm 0.15 \pm 0.15 \times 10^{-5}$ \\
$\sin \theta$ & $-0.42 \pm 0.15 \pm 0.15 \times 10^{-5}$ & 2.8 & $\cos 2 \theta$ & 3.2 \\
$\sin 2 \theta$ & $0.18 \pm 0.15 \pm 0.15 \times 10^{-5}$ & 1.2 & $\cos 3 \theta$ & $0.86 \pm 0.15 \pm 0.15 \times 10^{-5}$ \\
$\sin 3 \theta$ & $0.12 \pm 0.15 \pm 0.15 \times 10^{-5}$ & 0.8 & $\cos 4 \theta$ & $0.00 \pm 0.15 \pm 0.15 \times 10^{-5}$ \\
$\sin 4 \theta$ & $-0.02 \pm 0.15 \pm 0.15 \times 10^{-5}$ & 0.2 & $\cos 5 \theta$ & $0.17 \pm 0.15 \pm 0.15 \times 10^{-5}$ \\
$\sin 5 \theta$ & $-0.06 \pm 0.15 \pm 0.15 \times 10^{-5}$ & 0.4 & $\cos 6 \theta$ & $0.28 \pm 0.15 \pm 0.15 \times 10^{-5}$ \\
$\sin 6 \theta$ & $-0.13 \pm 0.15 \pm 0.15 \times 10^{-5}$ & 0.9 & $\cos 7 \theta$ & $0.18 \pm 0.15 \pm 0.15 \times 10^{-5}$ \\
$\sin 7 \theta$ & $-0.05 \pm 0.15 \pm 0.15 \times 10^{-5}$ & 0.3 & $\cos 8 \theta$ & $-0.23 \pm 0.15 \pm 0.15 \times 10^{-5}$ \\
& & $90^{\circ}$ rotation sequence & $0.18 \pm 0.11 \pm 0.11 \times 10^{-5}$ \\
Constant & $56.79 \pm 0.14 \pm 0.14 \times 10^{-5}$ & 405.7 & $\cos \theta$ & 2.6 \\
$\sin \theta$ & $0.10 \pm 0.20 \pm 0.20 \times 10^{-5}$ & 0.5 & & $-0.41 \pm 0.20 \pm 0.20 \times 10^{-5}$ \\
$\sin 2 \theta$ & $0.05 \pm 0.14 \pm 0.14 \times 10^{-5}$ & 0.4 & & 2.1 \\
\hline
\end{tabular}

The data suggest that the cosine coefficients contain small nonstatistical fluctuations. This is expected due to the choice of the alternating clockwise/counter-clockwise angular rotation sequence and phase. This sequence choice removes any slow linear components in the asymmetry from the sine coefficients of the fit. Such terms are not removed in the cosine terms and evidence of this can be seen in Table IV. The data also show evidence of correlations from run to run. This is not unexpected, and can be modeled by techniques developed for analyzing time series data [36]. Using the autoregressive integrated moving average method we find that the data are well represented by the inclusion of a moving average term, and that the value and error in the $\sin 2 \theta$ coefficient are essentially unchanged from those determined from the uncorrelated least-squares analysis.

The fitting coefficients and their associated statistical errors and standard deviations are averaged to determine the value of the $\sin 2 \theta$ coefficient for the combined set of data,

$$
a_{2}=1.1 \pm 1.0 \pm 1.0 \times 10^{-6} \text {. }
$$

The first value corresponds to the central value of the measurement, the second value corresponds to the statistical error, and the third value corresponds to the total error or standard deviation. The $\chi^{2}$ per degree of freedom is 0.9994 , indicating no significant random errors other than those associated with counting statistics. Since the two errors are equal within the limits of this measurement, only one error will be used in further discussions.

The final step in the analysis procedure is the calculation of the spin-correlation coefficient $A_{5}$. The spin-correlation coefficient is given by

$$
A_{5}=\frac{a_{2}(1+\phi)}{\widetilde{t_{10}} \widetilde{t_{20}} n \sigma_{0}},
$$

where $\phi$ represents the background of $\gamma$ rays. Using the experimental parameters summarized in Table $\mathrm{V}$, the spincorrelation coefficient is

$$
A_{5}=8.6 \pm 7.7 \times 10^{-6} \text {. }
$$

This value is consistent with time reversal invariance and sets a bound at the $95 \%$ confidence level of

$$
A_{5} \leqslant 2.2 \times 10^{-5} \text {. }
$$

\section{RESULTS AND CONCLUSIONS}

In order to compare our measurement to results in other systems, the spin-correlation coefficient $A_{5}$ must be converted into a more fundamental quantity. Simonius investigated PC TRNI [13] and showed that it can arise only through charged vector meson exchanges, with the lowestorder contribution arising from the charged $\rho$ exchange. The potential is given by $[13,14]$

$$
\begin{gathered}
V_{1,2}^{\rho}=\mathcal{V}_{1,2}^{\rho}\left[\tau_{1} \times \tau_{2}\right]_{3} \\
\mathcal{V}_{1,2}^{\rho}=\frac{m_{\rho}^{3} g_{\rho}^{2} \bar{g}_{\rho} \mu_{v}}{4 \pi M^{2}} \frac{e^{-m_{\rho} r_{12}}}{m_{\rho}^{3} r_{12}^{3}}\left(1+m_{\rho} r_{12}\right)\left(\boldsymbol{\sigma}_{1}-\boldsymbol{\sigma}_{2}\right) \cdot l
\end{gathered}
$$

where $\mathbf{r}_{\mathbf{1 2}}=\mathbf{r}_{\mathbf{1}}-\mathbf{r}_{\mathbf{2}}, l=\mathbf{r}_{\mathbf{1 2}} \times \frac{1}{2}\left(\mathbf{p}_{\mathbf{1}}-\mathbf{p}_{\mathbf{2}}\right), \mu_{v}=3.70 \mu_{n}$ is the isovector nucleon magnetic moment, $M$ is the nucleon mass, $g_{\rho}=2.79$ is the normal strong $\rho \mathrm{NN}$ coupling, and $\bar{g}_{\rho}$ is a dimensionless ratio of the PC TRNI coupling to $g_{\rho}$. Haxton et al. [1] and Engel et al. [14] have used this potential to explore PC TRNI effects in nuclear physics. In particular, Engel et al. calculated a PC TRNI optical potential to ana-

TABLE V. A summary of the experimental parameters in the time-reversal measurement. The target thickness $n$ is calculated using Monte-Carlo techniques. The unpolarized cross section $\sigma_{0}$ is taken from the optical model predictions of Ref. [37].

\begin{tabular}{lc}
\hline \hline$a_{2}$ & $1.1 \pm 1.0 \times 10^{-6}$ \\
$\phi$ & $6.38 \pm 0.01 \%$ \\
$\widetilde{t}_{10}$ & $0.67 \pm 0.05$ \\
$\widetilde{t}_{20}$ & $0.62 \pm 0.03$ \\
$n$ & $0.065 \mathrm{~b}^{-1}$ \\
$\sigma_{0}$ & $5.14 \mathrm{~b}$ \\
\hline \hline
\end{tabular}




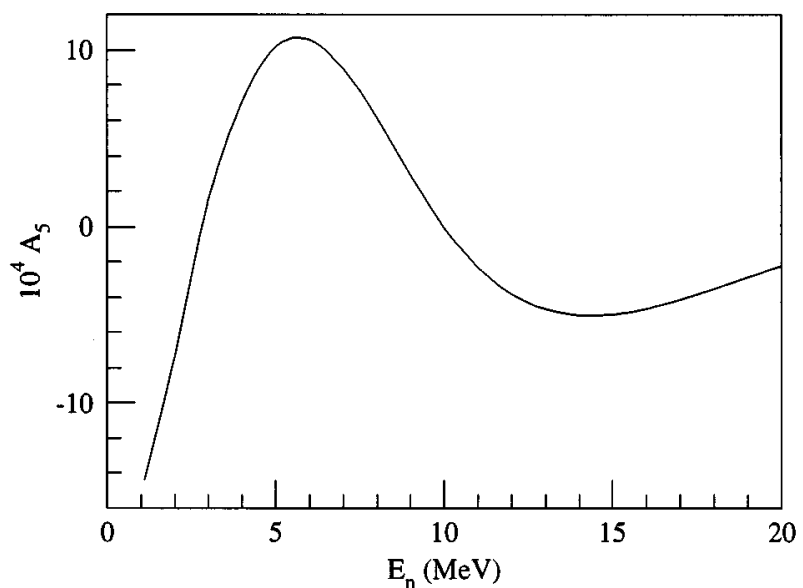

FIG. 7. The $T$-violating observable $A_{5}\left(\overline{g_{\rho}}=1\right)$ as a function of neutron energy [14].

lyze fivefold correlation data. Using the nucleon-nucleon potential, a spin-dependent one-body potential is constructed which can be used to calculate the quantity $A_{5}$ from coupled channels calculations. Taking $\bar{g}_{\rho}=1$ yields the results shown in Fig. 7.

Using this framework and our value of $A_{5}$,

$$
A_{5}=8.6 \pm 7.7 \times 10^{-6},
$$

a bound on $\overline{g_{\rho}}$ is extracted:

$$
\overline{g_{\rho}}=2.3 \pm 2.1 \times 10^{-2} \text {. }
$$

The fivefold measurement therefore gives a direct bound on the quantity $\bar{g}_{\rho}$.

Traditionally, limits on time-reversal violation are given in terms of $\alpha_{T}$, the ratio of $T$-violating to $T$-conserving nuclear matrix elements. The connection between $\alpha_{T}$ and $\overline{g_{\rho}}$ has been established by Haxton et al. [1] and is given by

$$
\alpha_{T}=\frac{3.6}{300} \overline{g_{\rho}}
$$

The scaling factor is determined by taking the ratio of the average $T$-violating nuclear matrix element from the Simonius potential to that of the strong interaction. For comparison purposes, our value of $\overline{g_{\rho}}$ is converted into $\alpha_{T}$, yielding

$$
\alpha_{T}=2.8 \pm 2.5 \times 10^{-4} \text {. }
$$

These values thus set bounds on time-reversal violation at the $95 \%$ confidence level of $\bar{g}_{\rho} \lesssim 5.9 \times 10^{-2}$ or $\alpha_{T} \lesssim 7.1 \times 10^{-4}$. Using these numbers, we now compare our measurement of time-reversal invariance to those in other systems. We consider bounds from both direct as well as indirect measurements.

Previously, the detailed balance measurement of Blanke et al. [10] had provided the most stringent direct test of parity-conserving, time-reversal invariance. The experiment tested detailed balance through differential cross section measurements of the reactions ${ }^{27} \mathrm{Al}(p, \alpha){ }^{24} \mathrm{Mg}$ and ${ }^{24} \mathrm{Mg}(\alpha, p){ }^{27} \mathrm{Al}$. Measurements were performed in the resonance region $(\sim 10 \mathrm{MeV})$ and the differential cross sections for the two reactions agreed to within $\Delta=\left(\sigma_{p, \alpha}-\sigma_{\alpha, p}\right) /\left(\sigma_{p, \alpha}+\sigma_{\alpha, p}\right) \lesssim 5.1 \times 10^{-3} \quad(80 \% \quad$ confidence).

The experimental observable $\Delta$, is not as simply related to the underlying nucleon-nucleon $T$-violating interaction as in the FC experiment. Numerous statistical analyses $[6,10,38-$ 40] have been performed to relate $\Delta$ to either $\alpha_{T}$ or to a third quantity $\xi$ (the ratio of $T$-violating to $T$-conserving amplitudes in the reaction). The comparison of our value of $\bar{g}_{\rho}$ to the detailed balance measurements depends heavily on which analysis is chosen.

The most complete analyses were presented by Harney et al. [38], $\xi=1.25 \times 10^{-3}(80 \%)$ and by Bunakov et al. [41] $\xi=1.33 \times 10^{-3}(80 \%)$. However, these analyses gain a factor of $1 / n$ in the precision of the variance by grouping data points that lie within a given correlation length into a single measurement. Effectively, this is treating the data as $n$ independent measurements, and the typical values of $n$ are 26 to 44 . This grouping procedure is not valid. When energy averages are considered, the $T$-violating observable is the variance of the deviation of the cross section from zero [42]. Independent measurements at the same precision cannot be used to lower the variance, but serve only to raise the confidence level of the bound. We therefore compare our results ${ }^{2}$ to the earlier bounds set by French et al. $\alpha_{T}=3.5 \times 10^{-3}$ (99\%) [6] and Boosé et al. $\alpha_{T}=2.7 \times 10^{-3}$ (80\%) [39]. Using these for comparison, our measurement represents an improvement of a factor of 4-5 over the detailed balance measurements of Blanke et al. [10].

A second set of measurements that set constraints on the $P$-conserving, $T$-violating interaction are the $P$-non-conserving, $T$-violating electric dipole moment (edm) measurements. Haxton et al. [1] have identified three mechanisms whereby $P$-non-conserving, $T$-violating observables can be generated through weak corrections to $P$-conserving, $T$-violating interactions. Measurements of the neutron electric dipole moment $\left(d \leqq 8 \times 10^{-26} e \mathrm{~cm}\right.$, Refs. $[43,44])$ and the atomic electric dipole moment of ${ }^{199} \mathrm{Hg}$ $\left(d \lesssim 1.3 \times 10^{-27} e \mathrm{~cm}\right.$, Ref. [45]) are used to set constraints on $\alpha_{T}$.

The neutron edm bound on $\alpha_{T}$ depends inversely on the as of yet unknown $P$-violating $\pi \mathrm{NN}$ coupling constant $f_{\pi}$ via $\alpha_{T}=7.0 \times 10^{-12} / f_{\pi}[1]$. This term is predicted to be large in meson exchange models [46], but measurements in ${ }^{18} \mathrm{~F}$ have shown the value is small and consistent with zero $\left(f_{\pi} \leqslant 0.34 \times 10^{-6}\right)$ [47]. Using this value, one obtains a lower bound on $\alpha_{T}$ of $\alpha_{T} \geqslant 2 \times 10^{-5}$. This value is an order of magnitude more restrictive, but we note that without a lower bound on $f_{\pi}$, this value could be much larger.

The atomic edm measurement of ${ }^{199} \mathrm{Hg}$, on the other hand, sets a bound independent of $f_{\pi}$, that is $\alpha_{T} \lesssim 1.1 \times 10^{-4}$ at the $95 \%$ confidence level [1]. This bound is more restrictive than the bound set by the present measurement, but we emphasize it is not a bound on a dynamical process. The $P$-conserving, $T$-violating bounds set from di-

\footnotetext{
${ }^{2}$ In a similar comparison, Haxton et al. [1] chooses to compare their results to those set by French et al. [6].
} 
pole moment measurements are indirect bounds on $\alpha_{T}$ and are thus complementary to the bounds set by the present direct measurement.

Direct tests of parity-conserving, time-reversal invariance continue to be discussed, both at high energies $(0.5 \mathrm{GeV} / c)$ in few-nucleon systems, and at low energies in resonance reactions in medium mass and heavy nuclei. Measurements of the FC in $\vec{p}-\vec{d}$ scattering have been proposed for the new storage ring facility COSY at Jülich [48]. Fewnucleon systems allow for a clean theoretical interpretation, and first estimates indicate potentially an order of magnitude improvement in a direct test of PC TRNI [49]. Resonance tests hold the most promise for large enhancements, but are also theoretically the hardest to interpret. Both neutron and charged particle tests have been discussed [50,51]. An important consideration in such tests is the need to obtain data on more than just one resonance. This is a realistic goal for the FC test in holmium, and for traditional detailed balance tests in charged particle reactions. It continues to be an issue for $P$-violating TRNI neutron transmission tests, where to date only single isolated resonances have been identified in polarizable nuclei (for example, in ${ }^{139} \mathrm{La}$ ).

In summary, we have tested reciprocity in nuclear reactions by measuring a $T$-violating spin-correlation coefficient $A_{5}$ of $(8.6 \pm 7.7) \times 10^{-6}$ in polarized neutron transmission through nuclear-spin aligned holmium. The measurement corresponds to a bound on a $T$-violating meson coupling of $\bar{g}_{\rho} \leqslant 5.8 \times 10^{-2}(95 \%)$. This represents the most precise test of parity-conserving, time-reversal noninvariance in a dynamical process.

\section{ACKNOWLEDGMENTS}

We gratefully acknowledge the helpful discussions with E. David Davis and Sastry G. Pantula. This work was supported in part by the U.S. Department of Energy, Office of High Energy and Nuclear Physics, under Contracts No. DEFG09-88-ER40411 and DE-FG05-91-ER40619.
[1] W. C. Haxton, A. Höring, and M. J. Musolf, Phys. Rev. D 50, 3422 (1994).

[2] P. Herczeg, Hyperfine Interact. 75, 127 (1992).

[3] J. Engel, P. H. Frampton, and R. P. Springer, Phys. Rev. D 53, 5112 (1996).

[4] M. T. Ressell, J. Engel, and P. Vogel, Phys. Rev. C 53, 2546 (1996).

[5] N. K. Cheung, H. E. Hennikson, and F. Boehm, Phys. Rev. C 16, 2381 (1977).

[6] J. B. French, A. Pandey, and J. Smith, in Tests of Time Reversal Invariance in Neutron Physics, edited by N. R. Roberson, C. R. Gould, and J. D. Bowman (World Scientific, Singapore, 1987), pp. 80-99.

[7] A. L. Barabanov, E. I. Sharapov, V. R. Skoy, and C. M. Frankle, Phys. Rev. Lett. 70, 1216 (1993).

[8] C. A. Davis et al., Phys. Rev. C 33, 1196 (1986).

[9] T. S. Bhatia et al., Phys. Rev. Lett. 48, 277 (1982).

[10] E. Blanke, H. Driller, W. Glöckle, H. Genz, A. Richter, and G. Schrieder, Phys. Rev. Lett. 51, 355 (1983).

[11] J. E. Koster, E. D. Davis, C. R. Gould, D. G. Haase, N. R. Roberson, L. W. Seagondollar, W. S. Wilburn, and X. Zhu, Phys. Lett. B 267, 23 (1991).

[12] P. K. Kabir, Phys. Rev. Lett. 60, 686 (1988).

[13] M. Simonius, Phys. Lett. 58B, 147 (1975).

[14] J. Engel, C. R. Gould, and V. Hnizdo, Phys. Rev. Lett. 73, 3508 (1994).

[15] P. R. Huffman, N. R. Roberson, W. S. Wilburn, C. R. Gould, D. G. Haase, C. D. Keith, B. W. Raichle, M. L. Seely, and J. R. Walston, Phys. Rev. Lett. 76, 4681 (1996).

[16] V. P. Alfimenkov, V. N. Efimov, T. T. Panteleev, and Y. I. Fenin, Sov. J. Nucl. Phys. 17, 149 (1973).

[17] A. L. Barabanov, Sov. J. Nucl. Phys. 45, 597 (1987).

[18] C. R. Gould, D. G. Haase, N. R. Roberson, H. Postma, and J. D. Bowman, Int. J. Mod. Phys. A 5, 2181 (1990).

[19] V. Hnizdo and K. W. Kemper, Phys. Rev. Lett. 59, 1892 (1987).

[20] V. Hnizdo, Phys. Rev. C 50, 2639 (1994).
[21] V. Hnizdo and C. R. Gould, Phys. Rev. C 49, R612 (1994).

[22] D. R. Tilley, H. R. Weller, and G. M. Hale, Nucl. Phys. A541, 1 (1992).

[23] E. J. F. Briesmeister, MCNP-A General Monte Carlo $N$-Particle Transport Code, ccc-200/monp 4a, Radiation Shielding Information Center Computer Code Collection (Oak Ridge National Laboratory, 1993).

[24] W. C. Koehler, J. W. Cable, M. K. Wilkinson, and E. O. Wollan, Phys. Rev. 151, 414 (1966).

[25] K. S. Krane, in Low-Temperature Orientation, edited by N. J. Stone and H. Postma (North Holland, Amsterdam, 1986), pp. 31-112.

[26] J. E. Koster, C. R. Gould, D. G. Haase, and N. R. Roberson, Nucl. Instrum. Methods Phys. Res. A 313, 464 (1992).

[27] M. Krusius, A. C. Anderson, and B. Holmström, Phys. Rev. 177, 910 (1969).

[28] J. E. Koster, C. R. Gould, D. G. Haase, and N. R. Roberson, Phys. Rev. C 49, 710 (1994).

[29] L. Stodolsky, Phys. Lett. B 172, 5 (1986).

[30] P. K. Kabir, Phys. Rev. D 37, 1856 (1988).

[31] J. D. Bowman et al., in Fundamental Symmetries in Nuclei and Particles, edited by H. Henrickson and P. Vogel (World Scientific, Singapore, 1989), pp. 1-29.

[32] J. P. Soderstrum, C. R. Gould, D. G. Haase, L. W. Seagondollar, M. B. Schneider, and N. R. Roberson, Phys. Rev. C 38, 2424 (1988).

[33] A. H. Hussein, J. M. Cameron, S. T. Lam, G. C. Nelson, and J. Soukup, Phys. Rev. C 15, 233 (1977).

[34] M. A. Kelley, B. L. Berman, R. L. Bramblett, and S. C. Fultz, Phys. Rev. 179, 1194 (1969).

[35] W. H. Press, S. A. Teukolsky, W. T. Vetterling, and B. P. Flannery, Numerical Recipes in FORTRAN: The Art of Scientific Computing, 2nd ed. (Cambridge University Press, Cambridge, England, 1992).

[36] W. A. Fuller, Introduction to Statistical Time Series, 2nd ed. (Wiley, New York, 1996).

[37] V. McLane, C. L. Dunford, and P. F. Rose, Neutron Cross 
Section Curves (Academic, New York, 1988), Vol. 2.

[38] H. L. Harney, A. Hüpper, and A. Richter, Nucl. Phys. A518, 35 (1990).

[39] D. Boosé, H. L. Harney, and H. A. Weidenmüller, Phys. Rev. Lett. 56, 2012 (1986).

[40] D. Boosé, H. L. Harney, and H. A. Weidenmüller, Z. Phys. A325, 363 (1986).

[41] V. E. Bunakov, H. L. Harney, and A. Richter, Nucl. Phys. A560, 71 (1993).

[42] E. D. Davis, in Fundamental Symmetries and Nuclear Structure, edited by J. N. Ginocchoi and S. P. Rosen (World Scientific, Singapore, 1988), pp. 52-67.

[43] K. F. Smith et al., Phys. Lett. B 234, 191 (1990).

[44] I. S. Altarev et al., Phys. Lett. B 276, 242 (1992).

[45] J. P. Jacobs, W. M. Klipstein, S. K. Lamoreaux, B. R. Heckel, and E. N. Fortson, Phys. Rev. Lett. 71, 3782 (1993).

[46] B. Desplanques, J. F. Donaghue, and B. R. Holstein, Ann. Phys. (N.Y.) 124, 449 (1980).

[47] E. G. Adelberger and W. C. Haxton, Annu. Rev. Nucl. Part. Sci. 35, 501 (1985).

[48] P. D. Eversheim et al., in Polarization Phenomena in Nuclear Physics, edited by E. J. Stephenson and S. E. Vigdor (AIP, New York, 1995), p. 191.

[49] M. Beyer, Nucl. Phys. A560, 895 (1993).

[50] P. R. Huffman, C. M. Frankle, C. R. Gould, D. G. Haase, J. A. Harvey, N. R. Roberson, and L. W. Weston, Phys. Rev. C 54, 2051 (1996).

[51] Time Reversal Invariance and Parity Violation in Neutron Reactions, edited by C. R. Gould, J. D. Bowman, and Yu. P. Popov (World Scientific, Singapore, 1994). 